\title{
siRNA-based Analysis of the Abrogation of the Protective Function of Membrane-associated Catalase of Tumor Cells
}

\author{
GEORG BAUER
}

Institute of Virology, Medical Faculty, University Medical Center Freiburg, Freiburg, Germany

\begin{abstract}
Tumor cells, in contrast to non-malignant cells, show sustained expression of membrane-associated NADPH oxidase-1 and therefore generate extracellular superoxide anions and their dismutation product $\mathrm{H}_{2} \mathrm{O}_{2}$. In order to prevent intercellular reactive oxygen species/reactive nitrogen species (ROS/RNS)-dependent apoptosis-inducing signaling, tumor cells need to express membrane-associated catalase that interferes with $\mathrm{HOCl}$ and nitric oxide/peroxynitrite signaling. Catalase is attached to tumor cells through the activity of transglutaminase-2 and is prevented from superoxide anion-dependent inhibition through coexpression of membrane-associated superoxide dismutase. Therefore, specific inhibition of membraneassociated catalase should reactivate intercellular ROS/RNSdependent apoptosis-inducing signaling. These processes are analyzed here through small interfering RNA-mediated knockdown of essential signaling compounds. This allows to establish a rather comprehensive picture of intercellular ROS/RNS signaling that may be instrumental for future therapeutic approaches.
\end{abstract}

Malignant cells, in contrast to their non-malignant parental cells, show sustained expression of membrane-associated NADPH oxidase-1 (NOX1) and generate extracellular superoxide anions [(1), reviewed in reference (2)]. These are involved in the control of proliferation of these cells and the maintenance of their transformed state. However, they also selectively endanger the survival of malignant cells through their central involvement in $\mathrm{HOCl}$ and nitric oxide/peroxynitrite (NO/PON)-dependent intercellular apoptosis-inducing signaling pathways (2-7).

This article is freely accessible online.

Correspondence to: Georg Bauer, Abteilung Virologie, Institut für Medizinische Mikrobiologie und Hygiene, Hermann-Herder Strasse 11, D-79104 Freiburg, Germany. E-mail: georg.bauer@uniklinikfreiburg.de

Key Words: Small interfering RNA, catalase, superoxide dismutase, tumor progression, tumor cell apoptosis.
Tumor progression leads to a state that is defined by resistance to exogenous $\mathrm{H}_{2} \mathrm{O}_{2}(8,9)$. Resistance is based on the expression of membrane-associated catalase (10-12). Catalase prevents $\mathrm{HOCl}$ signaling through decomposition of $\mathrm{H}_{2} \mathrm{O}_{2}$ and interferes with NO/PON signaling through oxidation of NO and decomposition of PON (7, 10-12).

As superoxide anions have the potential to inhibit catalase through reduction of compound $\mathrm{I}\left(\mathrm{CATFe} \mathrm{IV}^{\mathrm{IV}} \mathrm{O}^{+}\right)$to the inactive compound II $\left(\mathrm{CATFe}^{\mathrm{IV}}=\mathrm{O}\right)(13-15)$, the close association of NOX1 and catalase also requires the presence of membrane-associated superoxide dismutase (SOD) in order to modulate the superoxide anion concentration below a point of interference with catalase activity $(6,16)$. The local concentration of SOD on the outer surface of tumor cells is not sufficient to completely block $\mathrm{HOCl}$ and NO/PON signaling, in contrast to the dominant inhibitory potential of catalase (16).

Membrane-associated catalase and SOD in combination with active NOX1 have been defined as promising specific targets for novel therapeutic approaches (17-19). To understand the various modes of catalase inhibition/ inactivation and the resultant reactivation of intercellular ROS/RNS-dependent apoptosis-inducing signaling seems to be essential for the optimization of novel antitumor strategies based on reactive oxygen species/reactive nitrogen species (ROS/RNS) signaling.

Therefore, several modes of inhibition/inactivation of catalase are analyzed here, using a small interfering ribonucleic acid (siRNA)-based approach that allows to focus on the essential signaling molecules and their interactions. The effects of selective inhibition of membrane-associated catalase or SOD by cell-impermeable neutralizing antibodies and of siRNA-based knockdown of extra- and intracellular catalase are studied. This approach is supplemented by the analysis of singlet oxygen-dependent inactivation of catalase after application of the natural, garlic-derived NO dixoxygenase (NOD) inhibitor diallyl disulfide (DADS). Inhibition of NOD by plant compounds was recently shown to cause a local increase in free NO and transient inhibition of catalase, followed by singlet oxygen generation through the reaction between $\mathrm{H}_{2} \mathrm{O}_{2}$ and PON (20). 
This study should allow for definition of the specific target function of membrane-associated catalase and SOD of tumor cells and the quality of the reactivated intercellular ROS/RNS-driven signaling pathways.

\section{Materials and Methods}

Materials. The NOX1 inhibitor 4-(2-aminoethyl)benzenesulfonyl fluoride (AEBSF), the catalase inhibitior 3-aminotriazole (3-AT), catalase from bovine liver, DADS, the singlet oxygen scavenger histidine, myeloperoxidase (MPO) from human leukocytes, the hydroxyl radical scavenger mannitol, the inhibitor of NO synthase (NOS) $\mathrm{N}$-omega-nitro-L-arginine methylester hydrochloride (LNAME), the $\mathrm{HOCl}$ scavenger taurine, and Mn-containing SOD from Escherichia coli were obtained from Sigma Aldrich (Schnelldorf, Germany).

Inhibitors of caspase-3 (Z-DEVD-FMK), caspase-8 (Z-IETDFMK) and caspase-9 (Z-LEHD-FMK) were obtained from R\&D Systems (Wiesbaden-Nordenstadt, Germany).

The PON decomposition catalyst 5-,10-,15-,20-tetrakis(4sulfonatophenyl)porphyrinato iron(III) chloride (FeTPPS) was obtained from Calbiochem/Merck Biosciences GmbH, Schwalbach, Germany.

The catalase mimetic EUK 134 [chloro([2,2'-[1,2ethanediylbis[(nitrilo-kN)methylidyne] $b i s[6-m e t h o x y p h e n o l a t o-$ кO]]]-manganese was from Cayman (Ann Arbor, Michigan, USA) and was obtained from Biomol (Hamburg, Germany).

The mechanism-based POD inhibitor 4-aminobenzoyl hydrazide $(\mathrm{ABH})$ was obtained from Acros Organics (Geel, Belgium).

Media for cell culture. Cells were kept in RPMI-1640 medium containing $10 \%$ fetal bovine serum (FBS). FBS was heated for 30 mins at $56^{\circ} \mathrm{C}$ prior to use. Medium was supplemented with penicillin $(40 \mathrm{U} / \mathrm{ml})$, streptomycin $(50 \mu \mathrm{g} / \mathrm{ml})$, neomycin $(10 \mu \mathrm{g} / \mathrm{ml})$, moronal $(10 \mathrm{U} / \mathrm{ml})$ and glutamine $(280 \mu \mathrm{g} / \mathrm{ml})$. All supplements were obtained from Biochrom, Berlin, Germany. Cell culture was performed in plastic tissue culture flasks. Cells were passaged once or twice weekly.

Cells. The gastric carcinoma cell line MKN-45 was purchased from the Deutsche Sammlung für Mikroorganismen und Zellkulturen (Braunschweig, Germany). Cells were grown in suspension, with some cells attaching to the plastic culture dish, in RPMI-1640, with $10 \%$ serum and supplements as described above. Care was taken to avoid cell densities below $300,000 / \mathrm{ml}$ and above $10^{6} / \mathrm{ml}$.

Knockdown by treatment with specific siRNAs. All siRNAs were obtained from Qiagen (Hilden, Germany). The siRNAs directed towards human targets are detailed in Table I, including the respective target sequences and the order information. The sequences of custom made siRNAs are presented in Table II.

siRNAs were dissolved in suspension buffer supplied by Qiagen at a concentration of $20 \mu \mathrm{M}$. Suspensions were heated at $90^{\circ} \mathrm{C}$ for $1 \mathrm{~min}$, followed by incubation at $37^{\circ} \mathrm{C}$ for $60 \mathrm{~min}$. Aliquots were stored at $-20^{\circ} \mathrm{C}$.

Before transfection, $88 \mu \mathrm{l}$ of medium without serum and without antibiotics were mixed with $12 \mu$ l Hyperfect solution (Qiagen) and the required volume of specific siRNA or control siRNA to reach the desired concentration of siRNA during transfection (the standard concentration $24 \mathrm{nM}$ required $1.2 \mu \mathrm{l}$ ). The mixture was vortexed for a few seconds and then allowed to sit for $10 \mathrm{~min}$. It was then gently and slowly added to $300,000 \mathrm{MKN}-45$ cells in $1 \mathrm{ml}$ RPMI-1640 medium containing $10 \%$ FBS and antibiotics (12-well plates). The cells were incubated at $37^{\circ} \mathrm{C}$ in $5 \% \mathrm{CO}_{2}$ for $24 \mathrm{~h}$. Transfected cells were centrifuged and resuspended in fresh medium at the required density before use.

Determination of the efficiency of siRNA-mediated knockdown. The siRNA transfection system as described above had been optimized to allow a reproducible transfection efficiency of more than $95 \%$ of the cells and to avoid toxic effects (Bauer, unpublished data).

The efficiency of specific knockdown of transforming growth factor $\beta 1$ (TGF $\beta 1$ ), its receptor TGF $\beta R$, protein kinase $\mathrm{C}$ zeta (PKCzeta), bcl-2 homologous antagonist/killer (BAK), bcl-2associated $\mathrm{X}$ protein (BAX), mitochondria-derived activator of caspases (DIABLO), voltage-dependent anion channel (VDAC), apoptosis protease activating factor (APAF), caspase3, caspase-8, caspase-9, FAS receptor (FASR), FAS receptor-associated death domain (FADD) and matrix metalloprotease-2 (MMP2) mRNA had been experimentally determined by the supplier, using real-time quantitative polymerase chain reaction and was found to be more than $90 \%$ for $5 \mathrm{nM}$ siRNA.

The efficiency of knockdown of NOX1, dual oxidase-1 (DUOX1), transglutaminase-2 (TGM2) and catalase was based on functional quantitative assays and was more than $90 \%(5,12)$. The efficiency of knockdown of inducible NO synthase (iNOS), acidic sphingomyelinase (SMAse) and mitochondrial SOD was demonstrated through complete block of apoptosis after knockdown.

Autocrine ROS/RNS-mediated apoptosis induction. When seeded at appropriate density as well as cell number, tumor cells (in the presence of an inhibitor or inactivator of their protective catalase) establish apoptosis-inducing intercellular ROS/RNS signaling. Assays were performed in 96-well plates with $100 \mu \mathrm{l}$ of complete medium per well and contained 12,500 MKN-45 cells per well. For the reactivation of intercellular ROS/RNS signaling, the assays received either $0-2,000 \mathrm{ng} / \mathrm{ml}$ neutralizing antibody directed towards human catalase (anti-CAT), or $0-200 \mathrm{mM}$ of the catalase inhibitor 3-aminotriazole (3-AT), or $0-2000 \mathrm{ng} / \mathrm{ml}$ neutralizing antibody directed towards human SOD (anti-SOD), or 0-128 $\mu \mathrm{M}$ diallyl disulfide (DADS). Alternatively, intercellular ROS/RNS signaling was reactived through pretreatment with 0-24 nM siRNA directed towards human catalase (siCAT) or transglutaminase-2 (siTGM) for $24 \mathrm{~h}$. All assays were performed in duplicate. The plates were incubated at $37^{\circ} \mathrm{C}$ in an atmosphere of $5 \% \mathrm{CO}_{2}$. The percentages of apoptotic cells were then determined after $3.5-4 \mathrm{~h}$ as described below. Where indicated, inhibitors were added at the following final concentrations: AEBSF: $100 \mu \mathrm{M}$, SOD: $100 \mathrm{U} / \mathrm{ml}, \mathrm{ABH}: 150 \mu \mathrm{M}$, taurine: $50 \mathrm{mM}$, FeTPPS: $20 \mu \mathrm{M}$, L-NAME: $2.4 \mathrm{mM}$, mannitol scavenger: $20 \mathrm{mM}$; catalase: $30 \mathrm{U} / \mathrm{ml}$, EUK-134: $10 \mu \mathrm{M}$, caspase-3 inhibitor: $50 \mu \mathrm{M}$, caspase- 8 and -9 inhibitors: $25 \mu \mathrm{M}$.

Determination of the percentage of apoptotic cells. The percentage of apoptotic cells was determined by inverted phase-contrast microscopy based on the classical criteria for apoptosis, i.e. nuclear condensation or fragmentation and membrane blebbing (20-23). At least $2 \times 200$ cells were scored for each point of measurement in duplicate assays. Statistical analysis. Assays were performed in duplicate, unless otherwise stated. The empirical standard deviation was calculated 
Table I. Target sequences for siRNAs directed towards human targets.

\begin{tabular}{|c|c|c|c|}
\hline siRNA & Target & Target sequence & Order information \\
\hline siCo & Control (no target) & AAT TCT CCG AAC GTG TCA CGT & Control siRNA \\
\hline siNOX1 & NADPH oxidase-1 & CCG ACA AAT ACT ACT ACA CAA & Custom made \\
\hline siDUOX1 & Dual oxidase-1 & CCA GTC TAA CAC CAC AAC TAA & Custom made \\
\hline siiNOS2 & Inducible NO synthase-2 & CTG GGC CGT GCA AAC CTT CAA & Custom made \\
\hline siTGF $\beta 1$ & Transforming growth factor $\beta 1$ & CAG CAT ATA TAT GTT CTT CAA & Hs_TGFB1_6_HP Validated \\
\hline siTGF $\beta R$ & Transforming growth factor $\beta$ receptor & TCG GTT AAT AAC GAC ATG ATA & Hs_TGFBR2_7_HP Validated \\
\hline siPKCzeta & Protein kinase $\mathrm{C}$ zeta & GAC CAA ATT TAC GCC ATG AAA & Hs_PRKCZ_6_HP Validated \\
\hline siSMase & Acidic sphingomyelinase & TGG AAT TAT TAC CGA ATT GTA & Hs_SMPD1_1_HP siRNA \\
\hline siBAK & BCL-2 homologous antagonist/killer & AAG CGA AGT CTT TGC CTT CTC & Hs_BAK1_5_HP Validated \\
\hline $\operatorname{siBAX}$ & BCL-2-associated X protein & CAG GGT TTC ATC CAG GAT CGA & Hs_BAX_10_HP Validated \\
\hline siDIABLO & Mitochondria-derived activator of caspases & AAT GCG TTG ATT GAA GCT ATT & Hs_DIABLO_5_HP Validated \\
\hline siVDAC & Voltage-dependent anion channel-2 & AAA ATA CAA GTG GTG TGA GTA & Hs_VDAC2_5_HP Validated \\
\hline siAPAF & Apoptosis protease activating factor & AAG AGC AGC TAT GCT GAT TAA & Hs_APAF1_15_HP Validated \\
\hline siCASP3 & Caspase-3 & CTG AGA TGG GTT TAT GTA TAA & Hs_CASP3_7_HP Validated \\
\hline siCASP8 & Caspase- 8 & AAG AGT CTG TGC CCA AAT CAA & Hs_CASP8_11_HP Validated \\
\hline siCASP9 & Caspase-9 & CAG TGA CAT CTT TGT GTC CTA & Hs_CASP9_7_HP Validated \\
\hline siFASR & FAS receptor & AAG GAG TAC ACA GAC AAA GCC & Hs_FAS_7_HP Validated \\
\hline siFADD & FAS receptor-associated death domain & CAG CGG GAT CTC GTA TCT TTA & Hs_FADD_9_HP Validated \\
\hline siMMP2 & Matrix metalloprotease-2 & CAG GCT CTT CTC CTT TCA CAA & Hs_MMP2_5_HP Validated \\
\hline siCAT & Catalase & CCG GAT CTC ACT TGG CGG CAA & Hs_CAT_4_HP siRNA \\
\hline siTGM & Transglutaminase-2 & CAC AAG GGC GAA CCA CCT GAA & Hs_TGM2_6_HP siRNA \\
\hline siGPX4 & Glutathione peroxidase- 4 & GTC GAT GAA GAT CCA ACC CAA & Hs_GPX4_4_HP siRNA \\
\hline siSODm & Mitochondrial superoxide dismutase & ATC GTT ATG CTG AGT ATG TTA & Custom made \\
\hline
\end{tabular}

and is shown in the figures. Absence of standard deviation bars for certain points indicates that the standard deviation was too small to be reported by the graphic program. Empirical standard deviations were calculated merely to determine how close the results were obtained in parallel assays within the same experiment and not with the intention of statistical analysis of variance. The Yates continuity corrected chi-square test was used for the statistical determination of significances.

For clarity, some figures use a logarithmic scale annotated with the actual concentration applied.

\section{Results}

Neutralizing, cell-impermeable antibodies directed towards catalase caused apoptosis induction of MKN-45 cells $(p<0.001)$, resulting in a similar optimum curve as recently described for 3-AT (11) (Figure 1). Catalase-binding, but non-neutralizing antibodies, as well as antibodies directed towards signaling-irrelevant components such as fibronectin, laminin or the EGFR, were without apoptosis-inducing effect (18; data not shown). As shown by the effect of specific siRNA-mediated knockdown, apoptosis induction triggered by anti-catalase required active NOX1 at all concentrations of antibodies $(p<0.001)$, and was completely dependent on iNOS in the lower concentration range of antibodies $(p<0.001)$. In contrast, DUOX1 seemed to dominantly control apoptosis induction at higher concentrations of antibodies $(p<0.001)$. As shown by the knockdown
Table II. Sequences of custom-made siRNAs directed towards human targets.

\begin{tabular}{ll}
\hline siRNA & Sequence information \\
\hline siNOX1 & \\
Sense: & r(GAC AAA UAC UAC UAC ACA A)dTdT, \\
Antisense: & r(UUG UGU AGU AGU AUU UGU C)dGdG \\
siDUOX1 & \\
Sense: & r(AGU CUA ACA CCA CAA CUA A)dTdT \\
Antisense: & r(UUA GUU GUG GUG UUA GAC U)dGdG \\
siiNOS2 & \\
Sense & r(GGG CCG UGC AAA CCU UCA A)dTdT \\
Antisense: & r(UUG AAG GUU UGC ACG GCC C)dAdG \\
siSODm & \\
Sense: & r(CGU UAU GCU GAG UAU GUU A)dTdT \\
Antisense: & r(UAA CAU ACU CAG CAU AAC G)dAdT \\
\hline
\end{tabular}

NOX1: NADPH oxidase-1; DUOX1: dual oxidase-1; iNOS2: inducible NO synthase-2; SODm: mitochondrial superoxide dismutase.

experiments, apoptosis induction in the presence of anticatalase seemed to require the activity of TGF $\beta 1(p<0.001)$, TGF $\beta \mathrm{R}(p<0.001)$ and PKC zeta $(p<0.001)$ (Figure 1B), SMase $(p<0.001)$, VDAC $(p<0.001)$ and mitochondrial SOD $(p<0.001)$ (Figure 1C), BAK $(p<0.001)$, DIABLO $(p<0.001)$ and APAF $(p<0.001)$ (Figure 1D), caspase-9 $(p<0.001)$ and caspase-3 $(p<0.001)$, but not that of the FAS receptor 


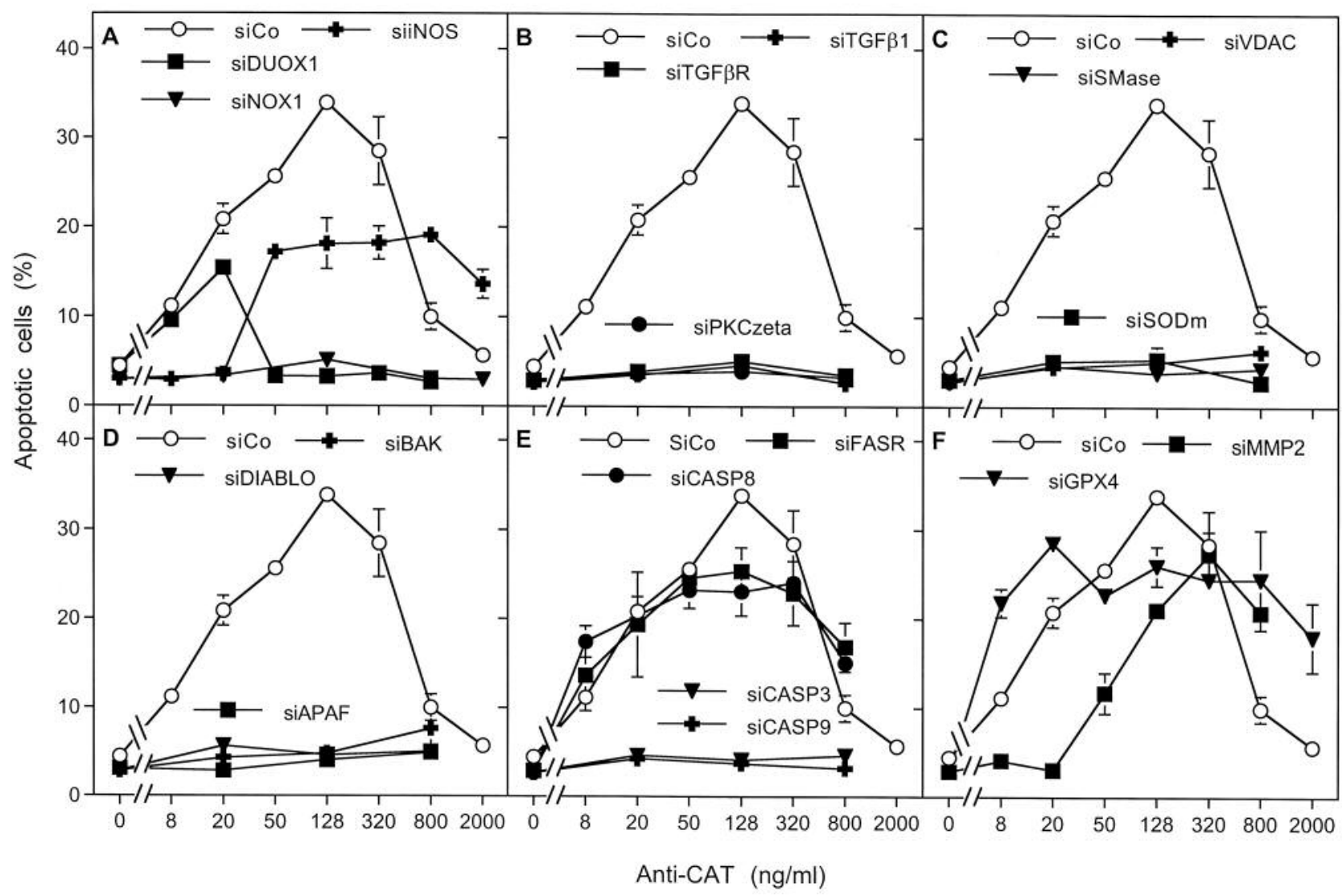

Figure 1. Small interfering ribonucleic acid (siRNA)-based analysis of reactivation intercellular reactive oxygen species/reactive nitrogen species $(R O S / R N S)$ signaling after selective inhibition of tumor cell-protective catalase through neutralizing antibodies directed towards membrane-associated catalase. Cells treated with control siRNA (siCo) or siRNAs directed towards inducible NO synthase (siiNOS), dual oxidase-1 (siDUOX1), NADPH oxidase 1 (siNOX1), transforming growth factor $\beta-1$ (siTGF $\beta 1$ ), TGF $\beta$ receptor (siTGF $\beta R$ ), protein kinase C zeta (siPKCzeta), voltage-dependent anion channel (siVDAC), acidic sphingomyelinase (siSMase), mitochondrial SOD (siSODm), bcl-2 homologous antagonist/killer (siBAK), mitochondriaderived activator of caspases (siDIABLO), FAS receptor (siFASR), caspases 3, 8 and 9 (siCASP3, 8 and 9), matrix metalloproteinase-2 (siMMP2) or glutathione peroxidase-3 (siGPX4) were challenged with increasing concentrations of neutralizing antibodies directed towards catalase (anti-CAT). Control antibodies directed towards laminin, fibronection and non-neutralizing antibody fragments had no apoptosis-inducing effect (data not shown).

(FASR) or of caspase-8 (Figure 1E). Knockdown of matrix metalloprotease-2 (MMP2) preferentially inhibited apoptosis induction in the lower concentration range of antibodies $(p<0.001)$, in line with the results recently described by Heinzelmann and Bauer (11) (Figure 1F). Knockdown of glutathione peroxidase 4 (GPX4) had a modulatory effect on apoptosis induction. These data show that selective inhibition of membrane-associated catalase of tumor cells caused intercellular ROS-dependent signaling and intracellular apoptosis executing pathways that were analogous to those found for 3-AT. Therefore, extracellular catalase seems to dominate the protective function. Its inhibition was sufficient to explain the previously determined effects of the catalase inhibitor 3-AT, which does, however, not allow differentiation between the effects of intra- and extracellular catalase.
If catalase were the sole and dominant target affected by either 3-AT or neutralizing monoclonal antibodies, siRNAmediated knockdown of catalase should have the same effect on tumor cells as treatment with 3-AT or antibodies. As shown in Figure 2, siRNA-mediated knockdown of catalase caused apoptosis induction dependent on the concentration of siRNA applied and in the mode of an optimum curve $(p<0.001)$. The effect was highly reproducible between three independent experiments (Figure 2A) and seemed to be based on reactivation of $\mathrm{NO} / \mathrm{PON}$ and $\mathrm{HOCl}$ signaling, as shown by the specific and consecutive inhibition by taurine (a selective inhibitor of $\mathrm{HOCl}$ signaling) $(p<0.001)$ and FeTPPS (a specific inhibitor of NO/PON signaling) $(p<0.001)$ (Figure 2B). Under optimal conditions of apoptosis induction after siRNAmediated knockdown of catalase (12 nM siCAT), intercellular apoptosis inducing signaling was mediated preferentially by the 


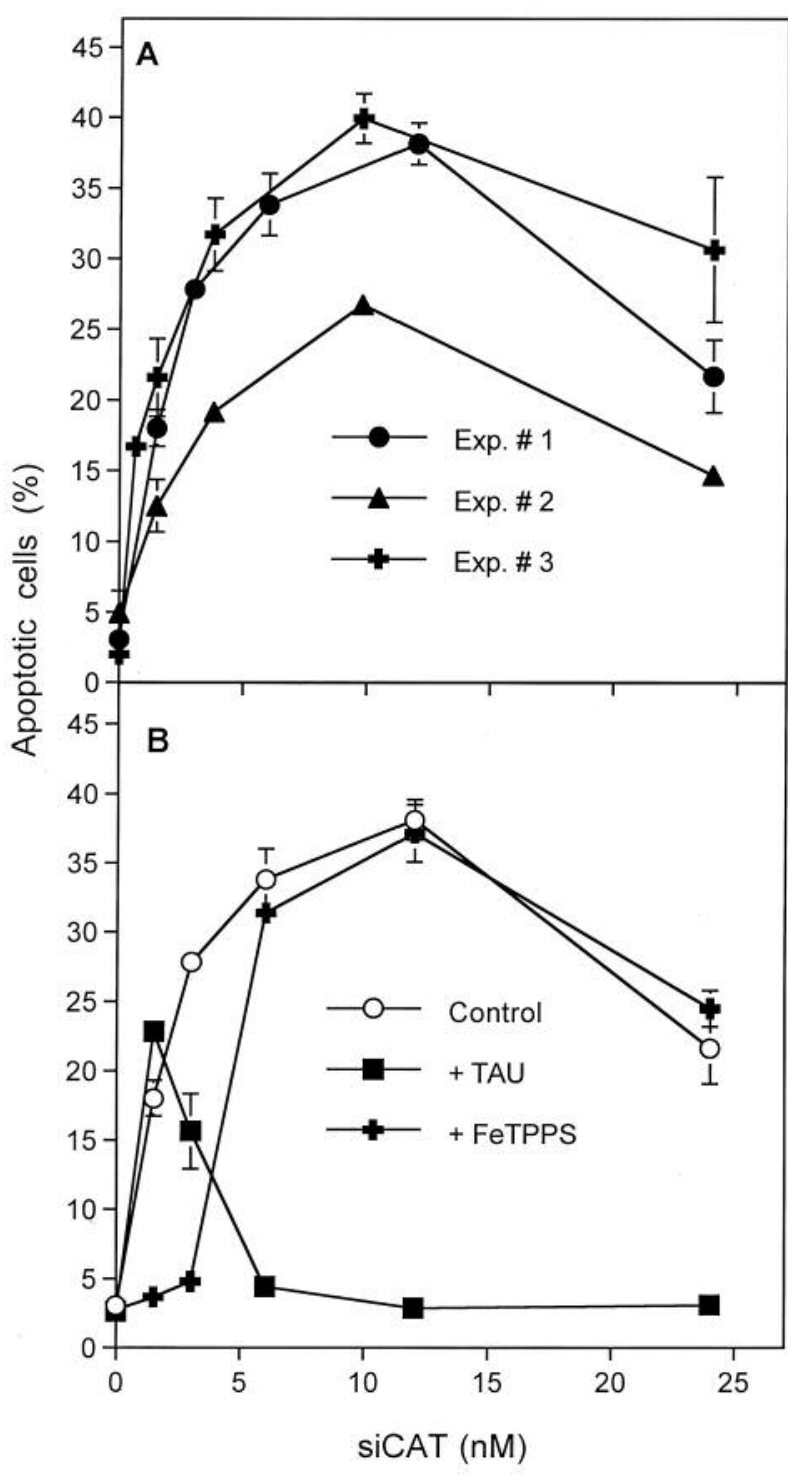

Figure 2. Small interfering ribonucleic acid (siRNA)-dependent knockdown of catalase reactivates intercellular apoptosis-inducing reactive oxygen species/reactive nitrogen species (ROS/RNS) signaling. A: Reproducibility of apoptosis induction in MKN-45 tumor cells by concentration-dependent siRNA-mediated knockdown of catalase as shown by three replicate experiments. B: siRNA-mediated knockdown of catalase causes initial reactivation of nitric oxide/peroxynitrite (NO/PON) signaling [inhibited by the PON decomposition catalyst 5 ,10-,15-,20-tetrakis(4-sulfonatophenyl)porphyrinato iron(III) chloride (FeTPPS)], followed by $\mathrm{HOCl}$ signaling [inhibited by the $\mathrm{HOCl}$ scavenger taurine (TAU)]. Exp.\#1,\#2,\#3:Experiment \#1, \#2, \#3; siCAT: siRNA directed towards human catalase.

HOCl signaling pathway, as knockdown of NOX1 $(p<0.001)$ and DUOXI $(p<0.001)$, but not of iNOS prevented apoptosis induction (Figure 3). The effect of DUOXI knockdown was compensated by addition of exogenous MPO $(p<0.001)$, thus

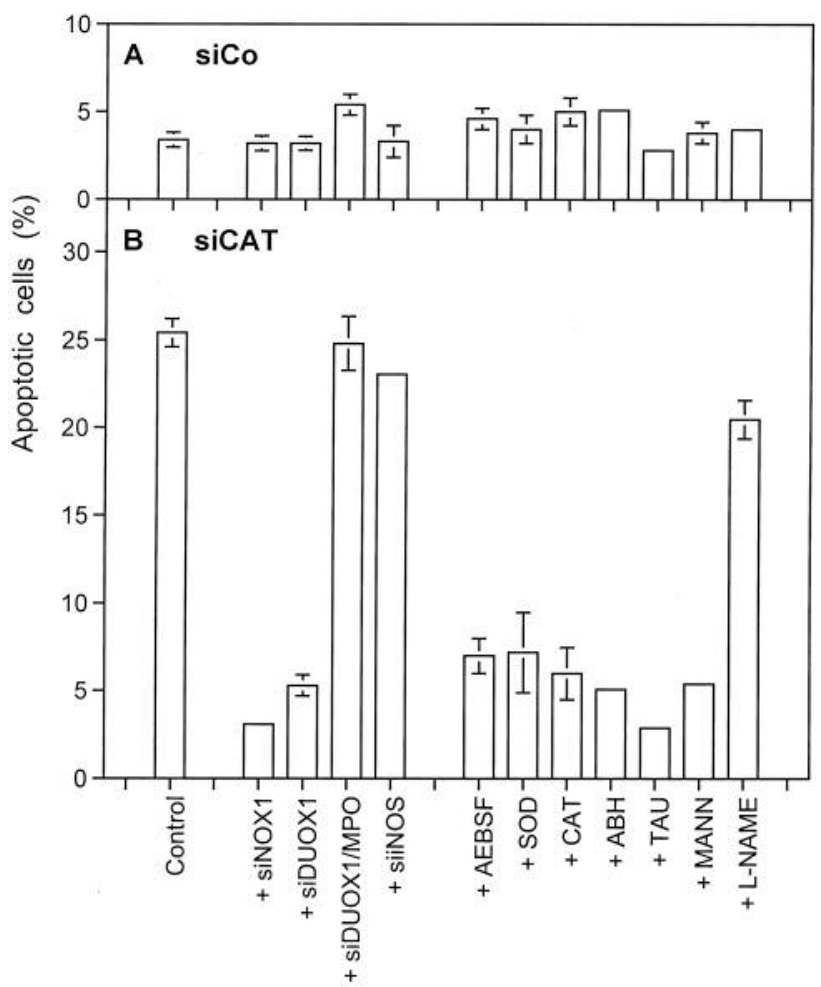

Figure 3. Reactive oxygen species (ROS)-dependent signaling after knockdown of catalase. A. Treatment of MKN-45 tumor cells with $12 \mathrm{nM}$ control siRNA (siCo) does not cause apoptosis induction. B. Knockdown by $12 \mathrm{nM}$ siRNA directed towards catalase (siCAT) reactivates apoptosis induction in MKN-45 cells. Apoptosis induction in siCAT-treated cells is abrogated by parallel knockdown of either NADPH oxidase1 (siNOX1) or dual oxidase-1 (siDUOX1), but not by knockdown of inducible NO synthase (siiNOS). The effect of siDUOX1 is abrogated through supplementation with exogenous myeloperoxidase (MPO). The effect of siCAT is inhibited by the NOX1 inhibitor 4-(2-aminoethyl)benzenesulfonyl fluoride (AEBSF), superoxide dismutase (SOD), catalase (CAT), the peroxidase inhibitor 4-aminobenzyoly hydrazide $(\mathrm{ABH})$, the $\mathrm{HOCl}$ scavenger taurine (TAU) and the hydroxyl radical scavenger mannitol (MANN), but not by the NO synthase inhibitor N-omega-nitro-L-arginine methylester hydrochloride (L-NAME).

confirming the role of enzymatic peroxidase function for DUOX during $\mathrm{HOCl}$ signaling. These conclusions were further substantiated by inhibitor studies, as inhibition of NOX1 by AEBSF $(p<0.001)$, scavenging of superoxide anions by SOD $(p<0.001)$, decomposition of $\mathrm{H}_{2} \mathrm{O}_{2}$ by catalase $(p<0.001)$, inhibition of peroxidase by $\mathrm{ABH}(p<0.001)$, scavenging of $\mathrm{HOCl}$ by taurine $(p<0.001)$ and scavenging of hydroxyl radicals by mannitol $(p<0.001)$ inhibited apoptosis induction, whereas inhibition of NOS by L-NAME had no significant inhibitory effect (Figure 3).

As the combination of the catalase inhibitor 3-AT and siRNA-mediated knockdown of catalase had an additive effect, resulting in a left-ward shift of the 3-AT-dependent 


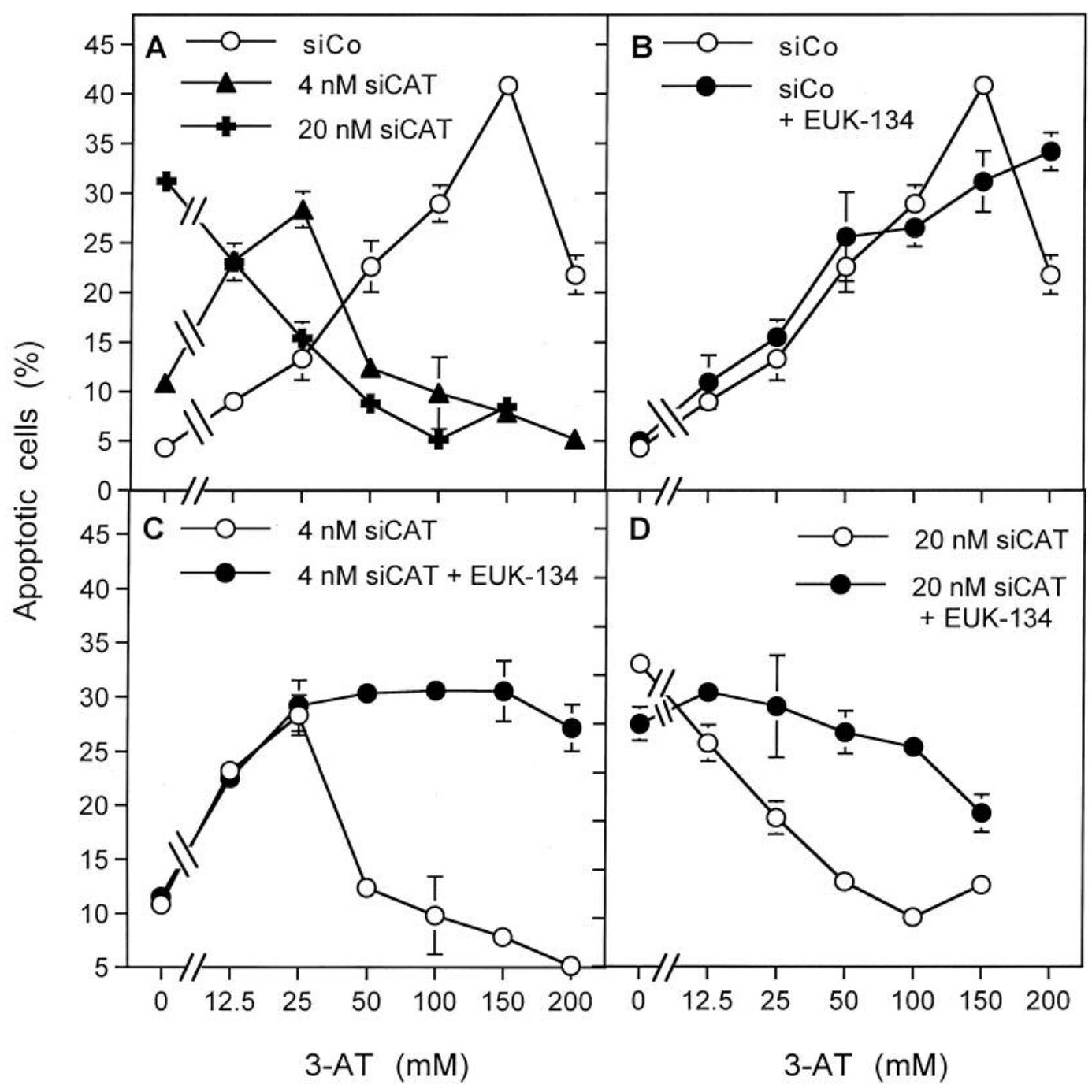

Figure 4. Cooperation between small interfering ribonucleic acid (siRNA)-mediated knockdown and 3-aminotriazol (3-AT)-mediated inhibition of catalase. A: 3-AT leads to an optimum of apoptosis induction in MKN-45 tumor cells. Additional siRNA-mediated knockdown of catalase (siCAT) leads to a leftwards shift of the optimum curve, indicating that siRNA-and inhibitor treatment act on the same target, i.e. catalase. B-D: Catalase mimetic [chloro([2,2'-[1,2-ethanediylbis[(nitrilo-kN)methylidyne]]bis[6-methoxyphenolato-kO]]]-manganese (EUK-134) at 0.25 $\mu M$ abrogates the supraoptimal inhibition of apoptosis induction, indicating that excess $\mathrm{H}_{2} \mathrm{O}_{2}$ causes the decline in apoptosis induction.

optimum curve of apoptosis induction $(p<0.001)$ (Figure $4 \mathrm{~A})$, it is clear that both treatments specifically target catalase. As addition of a low concentration of the catalase mimetic EUK-134 caused a shift from optimum curves to plateau-type curves $(p<0.001)$ (Figures 4B-D), excess $\mathrm{H}_{2} \mathrm{O}_{2}$ seems to be responsible for the supraoptimal inhibition of apoptosis induction.

If the effect of siRNA-mediated knockdown of catalase were solely due to catalase, its apoptosis signalingreactivating effect should be completely abrogated through the addition of exogenous catalase. Thereby, the simplest prediction was that the concentration of catalase required for abrogation of apoptosis induction should directly correlate with the concentration of siRNA applied. The resolution of this seemingly straightforward assumption exhibits, however, a certain degree of unexpected complexity. Transfection with $12 \mathrm{nM}$ siRNA directed towards catalase caused optimal induction of apoptosis $(p<0.001)$ that seemed to be mediated solely through the $\mathrm{HOCl}$ signaling pathway, as it was completely inhibited by taurine $(p<0.001)$ and not by FeTPPS (Figure 5A). Catalase at concentrations of up to $30 \mathrm{U} / \mathrm{ml}$ gradually inhibited apoptosis induction $(p<0.001)$, but higher concentrations of catalase caused an initial increase in apoptosis induction $(p<0.001)$ which was followed by 


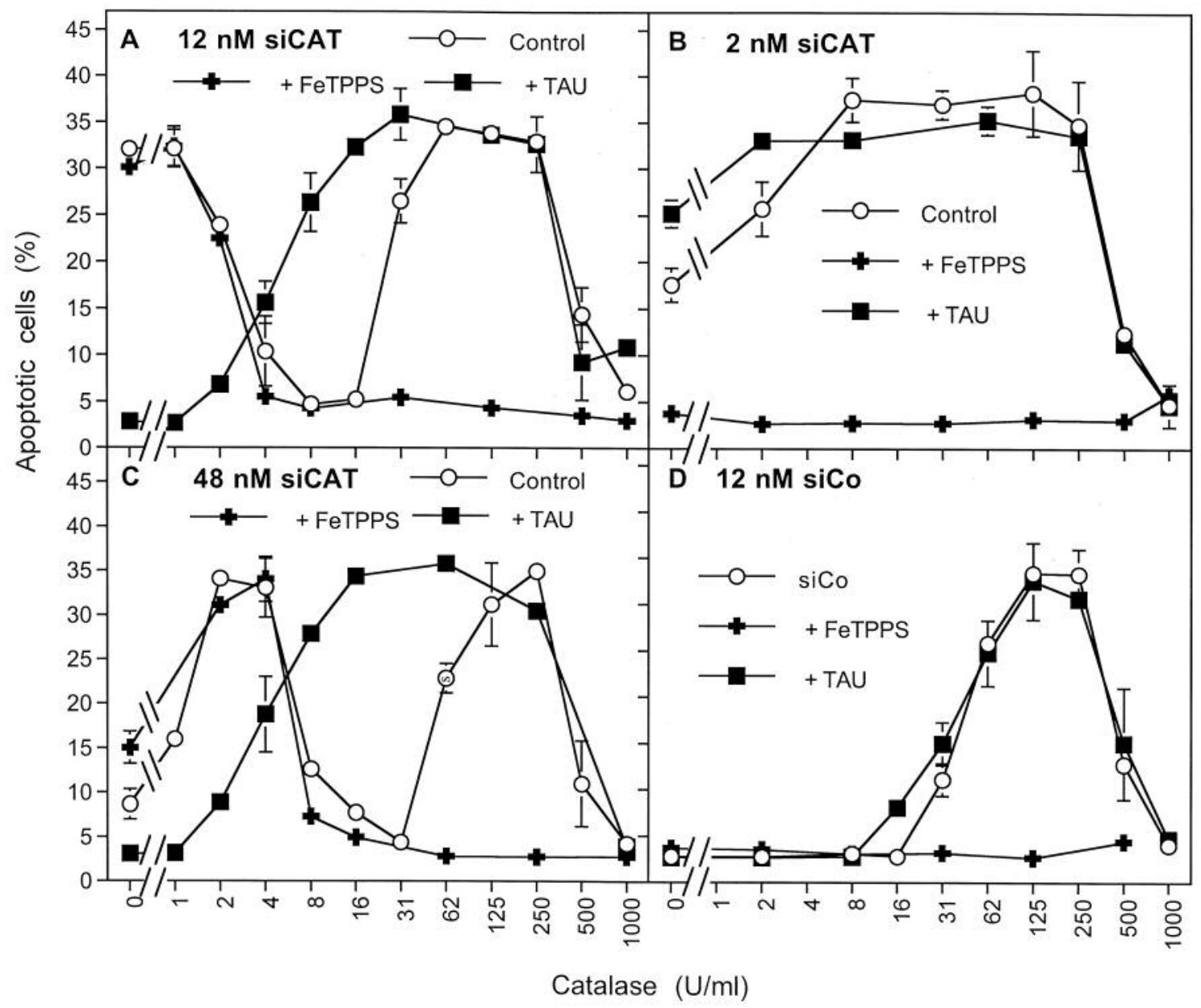

Figure 5. The effect of small interfering ribonucleic acid (siRNA)-mediated knockdown of catalase is abrogated by exogenous, soluble catalase. A: Treatment with $12 \mathrm{nM}$ siRNA directed towards catalase (siCAT) reactivates intercellular apoptosis-inducing signaling that is inhibited by taurine (TAU), but not by 5-,10-,15-,20-tetrakis(4-sulfonatophenyl)porphyrinato iron(III) chloride (FeTPPS). Addition of gradually increasing concentrations of catalase inhibit taurine-sensitive HOCl signaling and then allows FeTPPS-sensitive NO/peroxynitrite signaling, which is finally inhibited by very high concentrations of catalase. B: 2 nM siCAT causes NO/peroxynitrite signaling exclusively, which needs high concentrations of catalase for inhibition. C: Apoptosis induction after treatment with $48 \mathrm{nM}$ siCAT is due to HOCl signaling and is first enhanced by the addition of catalase and then follows the pattern shown in A. D: Control cells show NO/peroxynitrite-dependent apoptosis induction in the presence of medium to high concentrations of soluble catalase.

inhibition at very high concentrations $(p<0.001)$. The increase in apoptosis induction at catalase concentrations of between 15 and $500 \mathrm{U} / \mathrm{ml}$ seemed to be dependent on NO/PON signaling, as it was inhibited by FeTPPS $(p<0.001)$. Application of taurine at this concentration range of catalase caused a leftward shift of the curve $(p<0.001)$. This is explained by increased consumption of $\mathrm{H}_{2} \mathrm{O}_{2}$ by peroxidase, due to the scavenging of the reaction product $\mathrm{HOCl}$. As a consequence, $\mathrm{H}_{2} \mathrm{O}_{2}$-dependent interference with $\mathrm{NO} / \mathrm{PON}$ signaling was attenuated. When the rather low concentration of $2 \mathrm{nM}$ siRNA directed towards catalase was applied, the apoptosis-inducing effect $(p<0.001)$ was significantly lower than that obtained after treatment with $12 \mathrm{nM}$ siRNA, but the concentration of catalase required for abrogation of the apoptosis-inducing effect was dramatically higher $(p<0.001)$ than in the case of $12 \mathrm{nM}$ siRNA. Addition of exogenous catalase initially even increased apoptosis induction $(p<0.001)$. Very high concentrations of exogenous catalase were required to inhibit apoptosis induction mediated by the very low concentration of siRNA $(p<0.001)$. In contrast to apoptosis induction by $12 \mathrm{nM}$ siRNA, apoptosis after treatment with $2 \mathrm{nM}$ siCAT was solely dependent on $\mathrm{NO} / \mathrm{PON}$ signaling, as it was completely inhibited by the PON decomposition catalyst FeTPPS $(p<0.001)$, whereas the $\mathrm{HOCl}$ scavenger taurine did not have any effect (Figure 5B). Knockdown with $48 \mathrm{nM}$ siCAT seemed to reflect the situation 


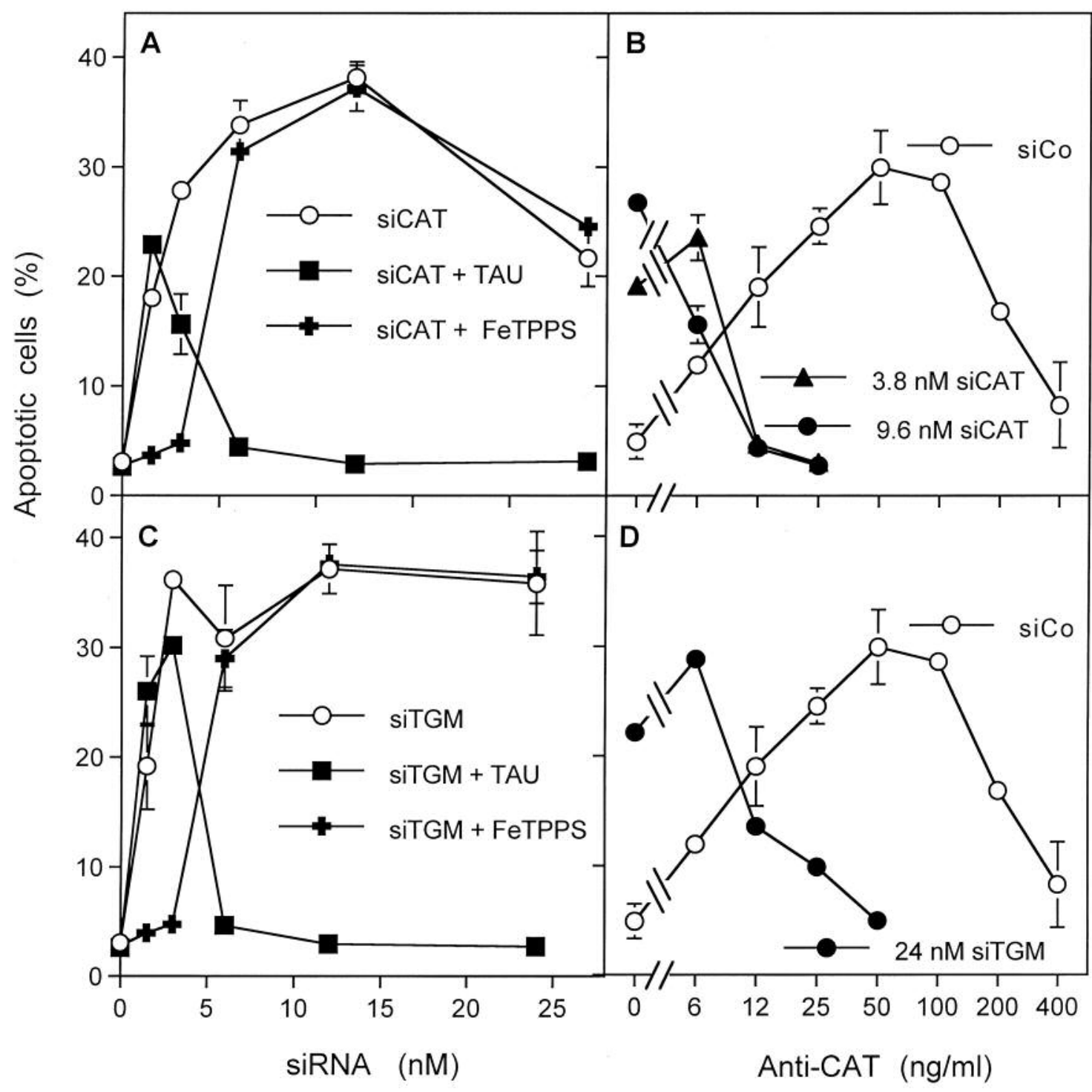

Figure 6. Small interfering ribonucleic acid (siRNA)-mediated knockdown of transglutaminase-2 (TGM) has an analogous effect as knockdown of catalase for MKN-45 cells. A, C: Analogous action of siRNA directed towards catalase (siCAT) or transglutaminase-2 (siTGM) through differential reactivation of NO/peroxynitrite signaling (inhibited by 5-,10-,15-,20-tetrakis(4-sulfonatophenyl)porphyrinato iron(III) chloride (FeTPPS) and subsequent HOCl signaling [inhibited by taurine (TAU)]. B, D: Additive effect of treatment with neutralizing antibodies directed towards catalase (Anti-CAT) and siRNA-mediated knockdown of catalase (siCAT) or transglutaminase (siTGM). Treatment of control siRNA (siCo)-transfected MKN45 tumor cells with anti-CAT results in an optimum curve of apoptosis induction. Additional knockdown of catalase (siCAT) (B) or TGM (siTGM) (D) causes a leftward shift of the optimum curve, indicating that identical target structures are affected.

at the right flank of the optimum curve, with apoptosis induction too low to be significant (Figure 5C). Addition of exogenous catalase brought apoptosis induction (mediated by $\mathrm{HOCl}$ signaling) to an optimal value $(p<0.001)$, before it was gradually inhibited by higher concentrations of catalase $(p<0.001)$. Catalase concentrations beyond $30 \mathrm{U} / \mathrm{ml}$ then increased apoptosis induction $(p<0.001)$ and very high concentrations of catalase were required for final inhibition of the process $(p<0.001)$. Interestingly, catalase addition to cells transfected with control siRNA caused apoptosis induction mediated by the NO/PON pathway, with an optimum at $125-250 \mathrm{U} / \mathrm{ml}(p<0.001)$ and final inhibition at $1,000 \mathrm{U} / \mathrm{ml}(p<0.001)$.

Knockdown of TGM2 had an analogous apoptosisinducing effect on MKN-45 tumor cells as knockdown of catalase $(p<0.001)$ (Figure 6A and C). This is explained by 


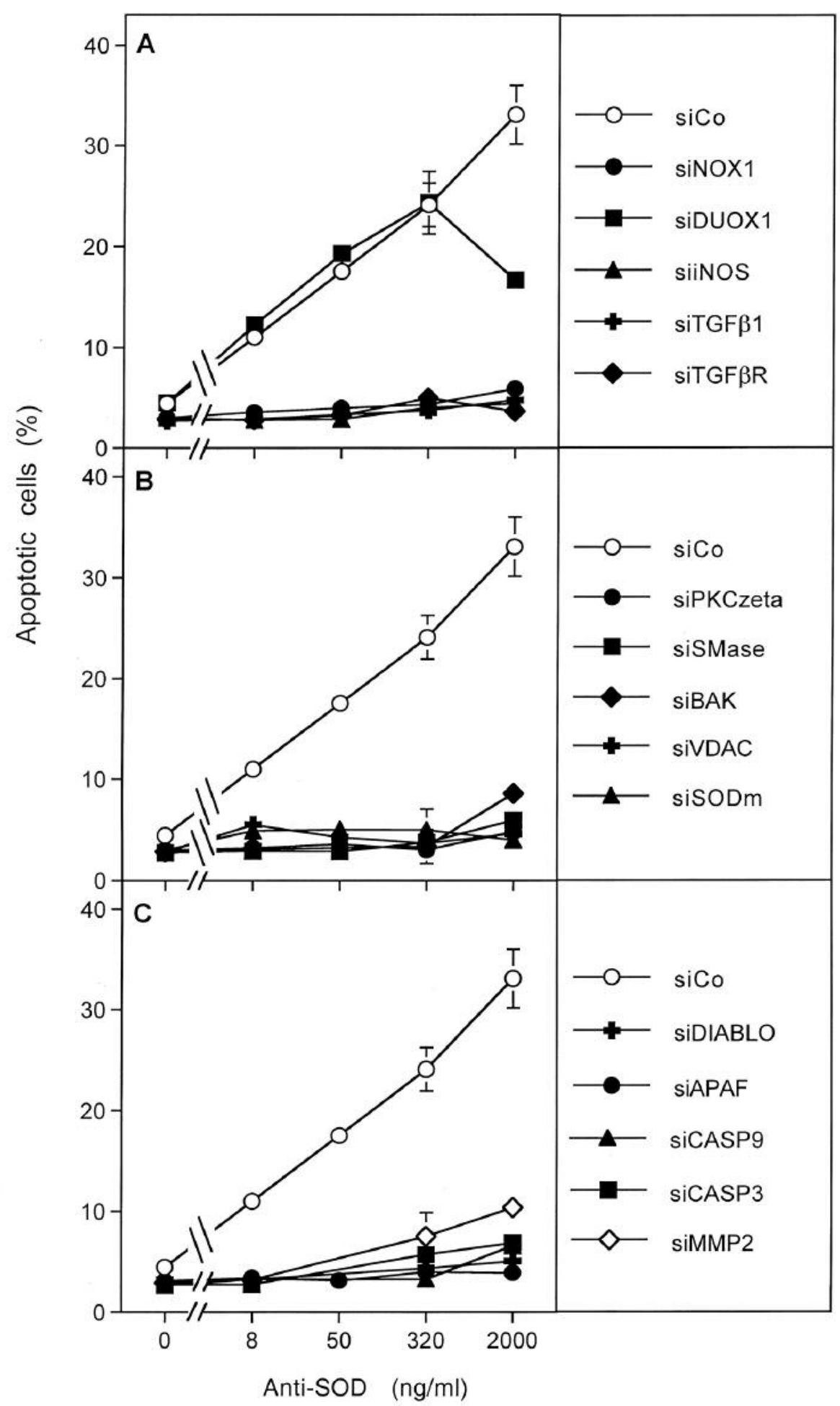

Figure 7. Small interfering ribonucleic acid (siRNA)-based analysis of apoptosis induction in MKN-45 cells by neutralizing antibodies directed towards superoxide dismutase (SOD). Antibodies directed towards SOD (anti-SOD) cause a linear increase in apoptosis induction in MKN-45 cells transfected with control siRNA (siCo). Apoptosis induction is completely inhibited in the case of pre-treatment with siRNA directed towards NADPH oxidase-1 (siNOX1), inducible NO synthase (siiNOS), transforming growth factor $\beta$-1 (siTGF $\beta 1$ ), TGF $\beta$ receptor (siTGF $\beta R$ ), protein kinase C zeta (siPKCzeta), acidic spingomyelinase (siSMASE), bcl-2 homologous antagonist/killer (siBAK), voltage-dependent anion channel (siVDAC), mitochondrial SOD (siSODm), mitochondria-derived activator of caspases (siDIABLO), apoptosis protease-activating factor APAF (siAPAF), caspase-3 and 9 (siCASP3 and -9) or matrix metalloprotease-2 (siMMP2). Knockdown of dual oxidase-1 (siDUOX1) only has a marginal inhibitory effect. 


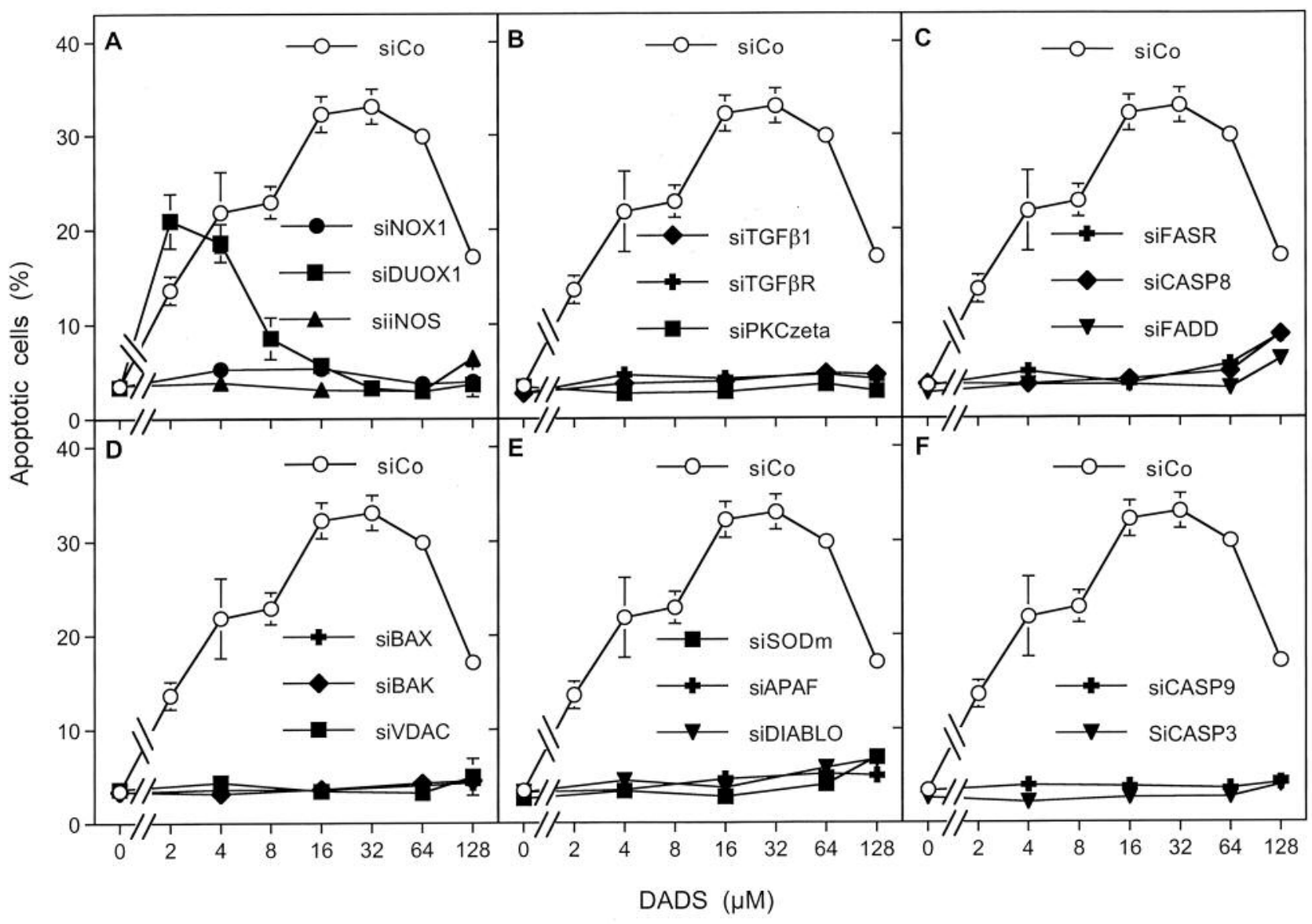

Figure 8. Small interfering ribonucleic acid (siRNA)-based analysis of apoptosis induction in MKN-45 cells after treatment with diallyl disulfide (DADS). Cells treated with control siRNA (siCo) or siRNAs directed towards NADPH oxidase 1 (siNOX1), dual oxidase-1 (siDUOX1), inducible NO synthase (siiNOS), transforming growth factor $\beta-1$ (siTGF $\beta 1$ ), TGF $\beta$ receptor (siTGF $\beta R$ ), protein kinase C zeta (siPKCzeta), FAS receptor (siFASR), caspase 8 (siCASP8), FASR-associated death domain (siFADD), bcl-2-associated X protein (BAX), bcl-2 homologous antagonist/killer (siBAK), voltage-dependent anion channel (siVDAC), mitochondrial superoxide dismutase (siSODm), apoptosis protease-activating factor APAF (siAPAF), mitochondria-derived activator of caspases (siDIABLO), caspases 3, and 9 (siCASP3, and 9) were challenged with increasing concentrations of DADS. The final outcome is analogous to the result shown in Figure 1 for neutralizing antibodies directed towards catalase, except that DADS-mediated apoptosis induction is inhibited by knockdown of FASR and caspase-8 by siFASR and siCASP8. This finding is in line with the inhibitory effect of knockdown of FADD by siFADD.

the abrogation of attachment of catalase to the membrane of tumor cells (12). Both treatments reactivated the same pattern of $\mathrm{NO} / \mathrm{PON}$ signaling $(p<0.001)$ that was followed by $\mathrm{HOCl}$ signaling at higher concentrations of the siRNAs applied $(p<0.001)$. The combination of either siCAT or siTGM treatment with neutralizing monoclonal antibodies directed towards catalase led to an analogous additive effect $(p<0.001)$ (Figure 6B and C), indicating that both siRNA treatments determined the concentration of membraneassociated catalase of tumor cells.

Neutralizing antibodies directed towards SOD caused apoptosis induction in a linear concentration-dependent mode $(p<0.001)$. Apoptosis induction in the presence of anti-SOD required the expression of NOX1 $(p<0.001)$, TGF $\beta 1$ $(p<0.001)$, TGF $\beta \mathrm{R}(p<0.001)$ and iNOS $(p<0.001)$. It was only marginally affected when DUOX1 was knocked down (Figure 7A). Apoptosis induction mediated by anti-SOD seemed to utilize PKCzeta $(p<0.001)$ and SMase $(p<0.001)$ and the mitochondrial pathway of apoptosis, as seen by the effects mediated by knockdown of $B A K(p<0.001), V D A C$ $(p<0.001)$, mitochondrial SOD $(p<0.001), \quad D I A B L O$ $(p<0.001)$ and caspase-9 $(p<0.001)$ (Figure 7B and C).

Inactivation of catalase through induction of cell-dependent extracellular production of singlet oxygen. Recent work from our group has shown that cell-derived, extracellular singlet 


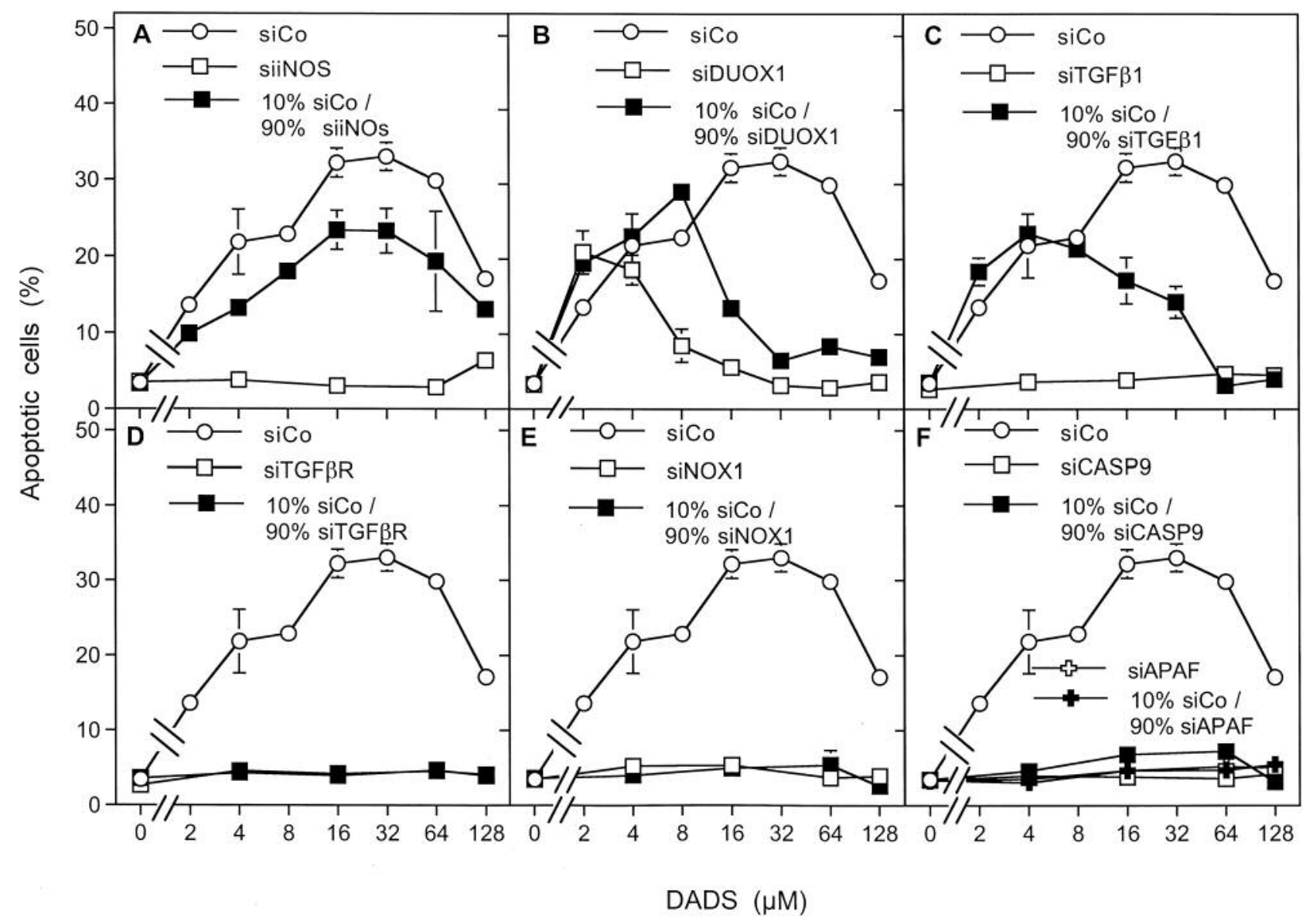

Figure 9. Detection of exchange of certain molecules between a minority of control tumor cells and a majority of tumor cells with knockdown of certain signaling molecules. A-C: Small interfering ribonucleic acid (siRNA)-mediated knockdown of inducible NO synthase (iNOS) and transforming growth factor $\beta-1$ (TGF $\beta 1$ ) causes complete abrogation of diallyl disulfide (DADS)-mediated apoptosis induction. Knockdown of dual oxidase-1 (siDUOX1) causes inhibition of apoptosis at medium and high concentrations of DADS. Addition of $10 \%$ cells treated with control siRNA (siCo) to cells treated with siiNOS, siDUOX1, siTGFB1 partially reconstitutes apoptosis induction and thus is indicative of transfer of NO, DUOX1-derived peroxidase domain (5) and TGF $\beta 1$ and between the cells. D-F: an analogous experiment, in which 10\% siCo-treated cells were added to cells with knockdown of TGF $\beta$ receptor (siTGF $\beta R$ ), NADPH oxidase 1 (siNOX1), caspase-9 (siCASP9) and apoptosis protease-activating factor (siAPAF) gives no indication of cross-supplementation.

oxygen inactivates catalase and thus reactivates intercellular apoptosis-inducing signaling $(7,17,20,24)$. Cell-derived singlet oxygen can be generated after enhancement of the intracellular NO concentration, followed by inhibition of membrane-associated catalase. This allows for subsequent interaction between peroxynitrous acid and $\mathrm{H}_{2} \mathrm{O}_{2}$, leading to the formation of singlet oxygen. This process is further amplified through the FASR and caspase $8(7,17,19,20)$. An ongoing study has shown that the garlic-derived compound DADS acts in an analogous mode to that of cyanidin, i.e. it inhibited NOD and thus modulated the endogenous NO concentration. This finally led to inactivation of protective catalase (Bauer, unpublished data). As shown in Figure 8, DADS caused apoptosis induction in
siCo-transfected MKN-45 cells dependent on its concentration and in the mode of an optimum curve $(p<0.001)$. Apoptosis induction was dependent on NOX1 $(p<0.001)$, iNOS $(p<0.001)$, TGF $\beta 1(p<0.001)$, TGF $\beta \mathrm{R}$ $(p<0.001)$ and PKC zeta $(p<0.001)$ over the whole concentration range of DADS, and on DUOX1 at its higher concentration range $(p<0.001)$ (Figure $8 \mathrm{~A}$ and $\mathrm{B})$. In contrast to apoptosis induction by direct catalase inhibitors, such as neutralizing antibodies, apoptosis induction by DADS required the action of the FASR $(p<0.001), \operatorname{FADD}(p<0.001)$ and caspase- $8(p<0.001)$ (Figure $8 \mathrm{C})$. The consequence of the signaling events triggered by these players was activation of the mitochondrial pathway of apoptosis $(p<0.001)$, as shown in Figure 8D-F. 


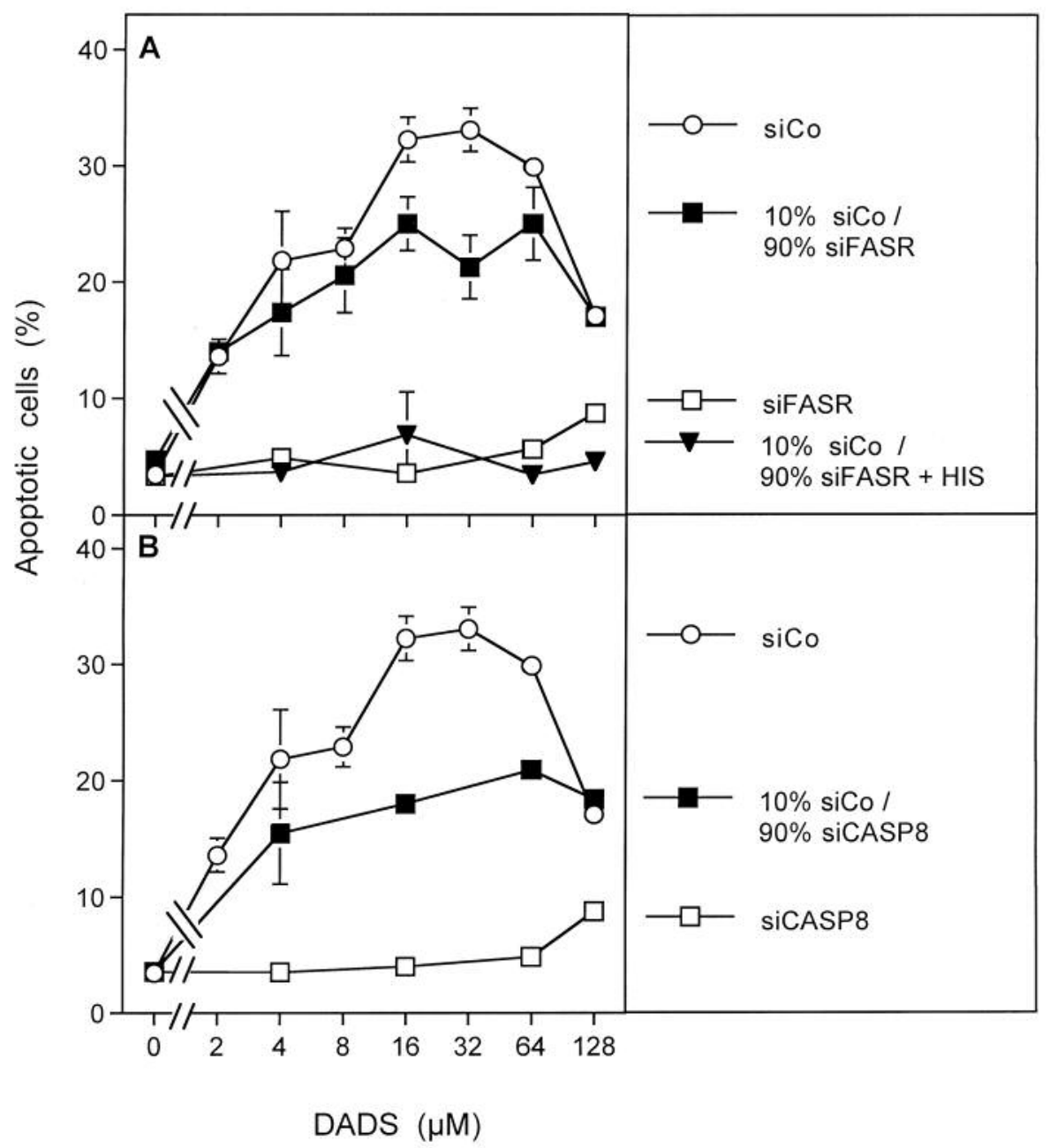

Figure 10. The role of FAS receptor (FASR) and caspase-8 in diallyl disulfide (DADS)-triggered intercellular signaling. Knockdown of FASR (siFASR) or caspase-8 (siCASP8) completely inhibits DADS-triggered apoptosis induction in MKN-45 tumor cells. Addition of 10\% control cells (siCo) to SiFASR- or siCASP8-treated cells partially restores apoptosis induction, indicative of cross-supplementation of FASR-dependent signaling molecules. The inhibition of cross-supplementation by the singlet oxygen scavenger histidine (HIS) points to a functional role of singlet oxygen.

The addition of $10 \%$ cells transfected with control siRNA to $90 \%$ of cells treated with either siiNOS, siDUOX1 or siTGF $\beta 1$ partially abrogated the inhibitory effect of the respective knockdown $(p<0.001)$ (Figure 9A-C), indicating that NO, DUOX1, and TGF $\beta 1$ act as as intercellular signaling effectors that seem to be exchanged between the minority of siCo-treated cells and the majority of cells with specific knockdown of relevant signaling components. In contrast, the addition of $10 \%$ siCo-transfected cells to cells with knockdown of TGF $\beta R$, NOX1 or caspase-9 did not counteract the inhibitory effect of knockdown (Figure $10 \mathrm{D}-\mathrm{F}$ ), indicating that the effector molecules defined by such siRNA-based knockdown acted strictly at the level of individual cells and were not shared within the cell population. This experimental approach allowed FASR-dependent enhancement of singlet oxygen generation to be differentiated from classical FASRdependent death receptor-mediated apoptosis induction. As shown in Figure 10A, the addition of 10\% siCo-transfected cells to cells with knockdown of the FASR nearly completely abrogated the strong inhibitory effect of siFASR $(p<0.001)$. The restoring effect of the minority of siCo-treated cells in this assay was abrogated through the singlet oxygen scavenger histidine $(p<0.001)$, pointing to the stringent connection between FASR-dependent enhancement of singlet oxygen generation. The combination of $10 \%$ siCo-treated cells with siCASP8-treated cells also caused strong interference with the inhibitory effect of caspase-8 knockdown $(p<0.001)$ (Figure $10 \mathrm{~B}$ ), pointing to the function of caspase- 8 in intercellular signaling processes and excluding that caspase- 8 was involved in the direct execution of cell death in this biological system. 

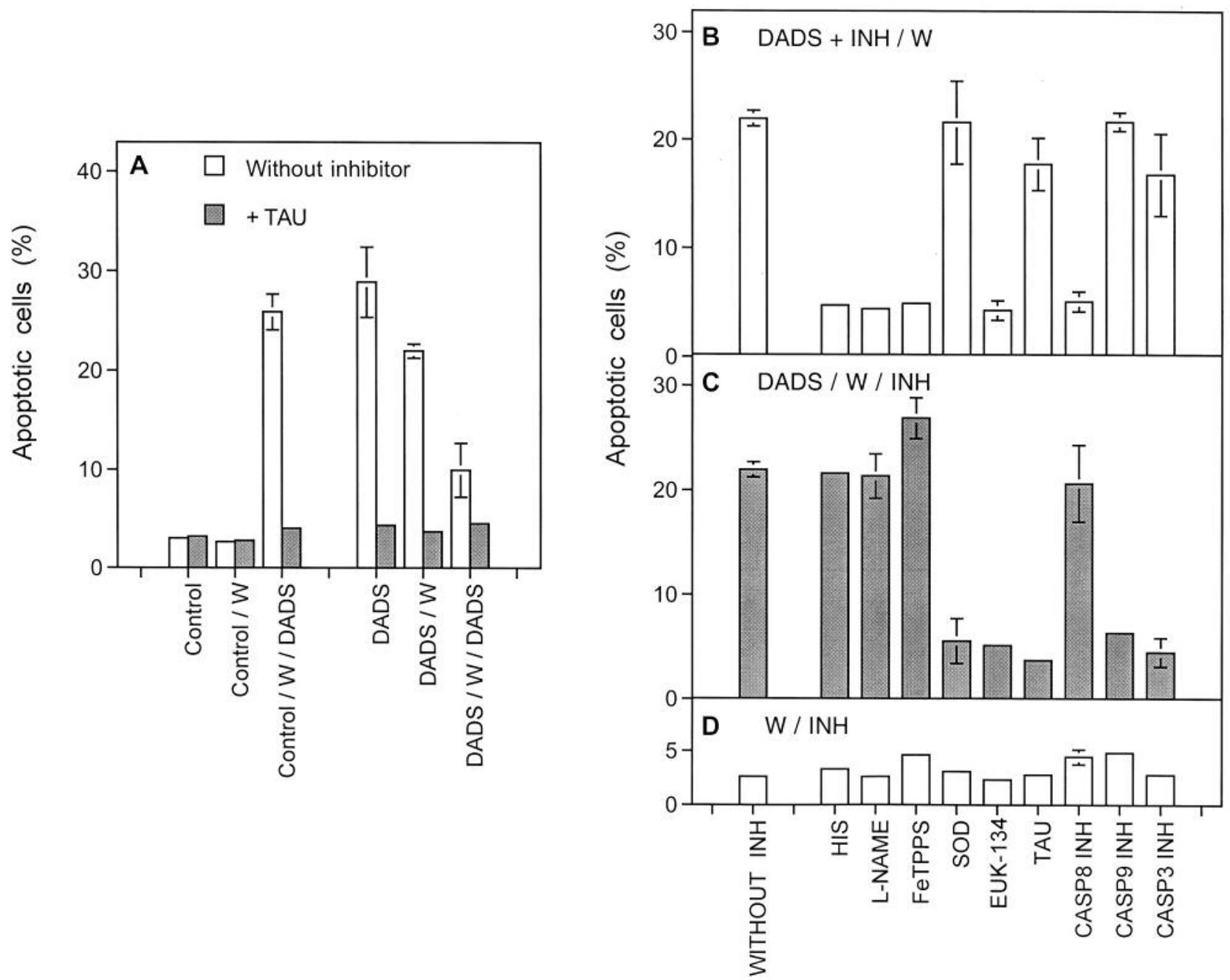

Figure 11. Apoptosis induction by diallyl disulfide (DADS) in tumor cells requires an early caspase-8-and singlet oxygen-dependent step. A: Pretreatment with $125 \mu \mathrm{M}$ DADS is sufficient for apoptosis induction. W: Removal of DADS and a two-fold washing step. B, C: The presence of the singlet oxygen scavenger histidine (HIS), the NO synthase (NOS) inhibitor N-omega-nitro-L-arginine methylester hydrochloride (L-NAME); the peroxynitrite decomposition catalyst 5-,10-,15-,20-tetrakis(4-sulfonatophenyl)porphyrinato iron(III) chloride (FeTPPS) or caspase-8 inhibitor (CASP8 INH) completely inhibits DADS-mediated apoptosis induction when the inhibitors (INH) are present during DADS treatment. Addition of these inhibitors after DADS treatment is without inhibitory effect. In contrast, superoxide dismutase (SOD), taurine, caspase -3, -8 and -9 inhibitors inhibit the apoptosis-inducing process that follows for DADS pre-treatment. D: No apoptosis induction is observed without treatment of the cells with DADS.

A short treatment with DADS, followed by a washing step and further incubation for several hours induced apoptosis nearly as efficient as observed in the continuous presence of DADS $(p<0.001)$ (Figure 11A). This is explained by an initial DADS-triggered, step which is followed by intercellular ROS-mediated signaling. Thereby, the initial step (induced by $125 \mu \mathrm{M}$ DADS) required the action of singlet oxygen, $\mathrm{NO}, \mathrm{PON}, \mathrm{H}_{2} \mathrm{O}_{2}$ and caspase- 8 , as it was inhibited by histidine $(p<0.001)$, L-NAME $(p<0.001)$, FeTPPS $(p<0.001)$, EUK-134 $(p<0.001)$ and caspase- 8 inhibitor $(p<0.001)$ (Figure 11B). In contrast, subsequent signaling through the $\mathrm{HOCl}$ signaling pathway was independent of singlet oxygen, $\mathrm{NO}, \mathrm{PON}$ and caspase- 8 , but required the action of superoxide anions $(p<0.001), \mathrm{H}_{2} \mathrm{O}_{2}$ $(p<0.001)$, $\mathrm{HOCl}(p<0.001)$, caspase-9 $(p<0.001)$ and caspase-3 $(p<0.001)$ (Figure 11C).

\section{Discussion}

These data comfirm that selective and gradual inhibition of membrane-associated catalase of tumor cells or siRNAmediated knockdown of the total catalase in tumor cells 
leads to an optimum curve of apoptosis induction, dependent on the strength of inhibition (11). The quality of signaling is similar in both approaches, i.e. NO/PON signaling is reactivated at lower degrees of catalase inhibition, preferential $\mathrm{HOCl}$ signaling at optimal inhibition and supraoptimal decline at higher degrees of inhibition. As this supraoptimal decline can be abrogated by well-balanced concentration of the catalase mimetic EUK-134, it is characterized as the negative effect of excess $\mathrm{H}_{2} \mathrm{O}_{2}$ on $\mathrm{HOCl}$ signaling, in line with previous analysis (25).

As addition of exogenous catalase was sufficient to abrogate the ROS/RNS-activating effects of siRNA-mediated knockdown of catalase, extracellular catalase seems to be the dominating factor for the control of intercellular ROS/RNS signaling. The characteristics of apoptosis induction in tumor cells by cell-impermeable antibodies or siRNA-mediated general knockdown of catalase are not only in good agreement with previous studies of 3-AT-dependent catalase inhibition $(11,26)$, but also allow focus to be placed on the specific role of membrane-associated catalase.

In the siRNA-based approach, it seemed to be paradox that the effect of the lowest concentration of siRNA applied required the highest concentration of exogenously added catalase for abrogation. This finding is explained by the specific features of NO/PON signaling, which was reactivated at the low concentration of siCAT. As NO/PON signaling takes place in ultimate vicinity of the membrane, its prevention by catalase requires a high local concentration of the enzyme (11). In tumor cells this is achieved by attachment of the catalase to the outside of the membrane. In the experimental situation, a high local concentration of soluble catalase can only be achieved when the overall concentration of the enzyme is very high.

Reactivation of intercellular ROS/RNS-dependent apoptosisinducing signaling by siRNA-mediated knockdown of transglutaminase is explained by lack of attachment of released catalase to the cell membrane (12) and therefore leads to an analogous signaling profile as direct inhibition of catalase.

In contrast, indirect inhibition of catalase through free superoxide anions after antibody-dependent inhibition of membrane-associated SOD preferentially causes NO/PONmediated apoptosis induction and a constant increase of apoptosis induction dependent on the concentration of antibodies, without an indication for an optimum curve. This finding is in agreement with the conclusion that inhibition of SOD not only sets free superoxide anions that inhibit catalase (13-15), but also interferes with enzymatic dismutation of superoxide anions to $\mathrm{H}_{2} \mathrm{O}_{2}$. Thus the substrate for peroxidasedependent $\mathrm{HOCl}$ signaling is lacking, furthermore, the counterbalancing effect of $\mathrm{H}_{2} \mathrm{O}_{2}$ towards $\mathrm{NO} / \mathrm{PON}$ signaling is abrogated.

In contrast to direct or indirect inhibition of catalase, its singlet oxygen-dependent inactivation triggered by the NOD inhibitor DADS shows a higher degree of complexity. Analogous findings have been reported for direct inactivation of catalase by exogenous singlet oxygen (24) and by cellderived singlet oxygen after modulation of the endogenous NO concentration through the NOD inhibitor cyanidin $(7,17$, 20). The effect of DADS requires an initial, autoamplificatory step of singlet oxygen generation and catalase inactivation, followed by intercellular ROS/RNS-dependent apoptosisinducing signaling. As superoxide anions, $\mathrm{H}_{2} \mathrm{O}_{2}$, $\mathrm{NO}$ and PON are involved in both steps, the knockdown of essential players is not suitable or adequate to unravel the complete signaling event. Rather, the combination of inhibition studies with siRNA-based analysis allows the complete picture to be painted. Like direct inhibition, singlet oxygen-dependent inactivation of catalase initially reactivates the $\mathrm{NO} / \mathrm{PON}$ signaling pathway, which is followed by the $\mathrm{HOCl}$ pathway at higher degrees of inactivation, finally leading to a suproptimal decline of apoptosis induction. Based on the siRNA profile, direct and indirect inhibition of catalase as well as its singlet oxygen-dependent inactivation are executed by the mitochondrial pathway of apoptosis. The addition requirement for the FASR and caspase- 8 as seen for DADSmediated apoptosis induction does not reflect the parallel activation of a death receptor-dependent apoptosis-inducing pathway, but rather the role of the FASR in the amplification of singlet oxygen generation $(7,17)$. This signaling function is well differentiated from direct cell death induction through the mixing experiments shown in Figure 9. As knockdown of FASR or caspase- 8 completely blocks apoptosis induction mediated by DADS, but $10 \%$ of siCO-treated control cells in a population of $90 \%$ siFASR- or siCASP8-treated cells strongly reconstitutes apoptosis induction, the direct effect is excluded and the intercellular signaling aspect is verified.

The knowledge of these signaling events can be utilized to establish synergistic effects that utilize the specific chemical biology of tumor cell-specific ROS and RNS signaling. It hopefully will be useful for the establishment and optimization of therapeutic approaches that work along these lines.

\section{Acknowledgements}

The Author thanks J. Brandel (Freiburg) for technical support and acknowledges support from RiscRad, EuroTransBio (ETB1 0315012B), Hans-Sauer-Stiftung and COST Action CM0603.

\section{References}

1 Irani K, Xia Y, Zweier JL, Sollott SJ, Der CJ, Fearon ER, Sundaresan $M$, Finkel $\mathrm{T}$ and Goldschmidt-Clermont PJ: Mitogenic signaling by oxidants in Ras-transformed fibroblasts. Science 275: 1649-1652, 1997.

2 Bauer G: Tumor cell protective catalase as a novel target for rational therapeutic approaches based on specific intercellular ROS signaling. Anticancer Res 32: 2599-2624, 2012. 
3 Bauer G: Targeting extracellular ROS signaling of tumor cells Anticancer Res 34: 1467-1482, 2014.

4 Bauer G: Increasing the endogenous NO level causes catalase inactivation and reactivation of intercellular apoptosis signaling specifically in tumor cells. Redox Biol 6: 353-371, 2015.

5 Herdener M, Heigold S, Saran M and Bauer G: Target cellderived superoxide anions cause efficiency and selectivity of intercellular induction of apoptosis. Free Radical Biol Med 29: 1260-1271, 2000

6 Heigold S, Sers C, Bechtel W, Ivanovas B, Schäfer R and Bauer G: Nitric oxide mediates apoptosis induction selectively in transformed fibroblasts compared to nontransformed fibroblasts. Carcinogenesis 23: 929-941, 2002.

7 Pottgiesser S, Heinzelmann S and Bauer G: Intercellular HOClmediated apoptosis induction in malignant cells: interplay between NOX1-dependent superoxide anion generation and DUOX-related HOCl-generating peroxidase activity. Anticancer Res 35: 5927-5943, 2015.

8 Deichman G: Natural selection and early changes of phenotype of tumor cells in vivo: Acquisition of new defense mechanisms. Biochem 65: 78-94, 2000.

9 Deichman G: Early phenotypic changes of in vitro transformed cells during in vivo progression: possible role of the host innate immunity. Sem Cancer Biol 12: 317-326, 2002.

10 Bechtel W and Bauer G: Catalase protects tumor cells against apoptosis induction by intercellular ROS signaling. Anticancer Res 29: 4541-4557, 2009.

11 Heinzelmann S and Bauer G: Multiple protective functions of catalase against intercellular apoptosis-inducing ROS signaling of human tumor cells. Biol Chem 391: 675-693, 2010.

12 Böhm B, Heinzelmann S, Motz M and Bauer G: Extracellular localization of catalase is associated with the transformed state of malignant cells. Biol Chem 396: 1339-1356, 2015.

13 Kono Y and Fridovich I: Superoxide radical inhibits catalase. J Biol Chem 257: 5751-5754, 1982.

14 Shimizu N, Kobayashi K and Hayashi K: The reaction of superoxide radical with catalase. Mechanism of the inhibition of catalase by superoxide radical. J Biol Chem 259: 4414-4418, 1984.

15 Fridovich I: Biological effects of the superoxide radical. Arch Biochem Biophys 247: 1-11, 1986.
16 Bauer G: HOCl-dependent singlet oxygen and hydroxyl radical generation modulate and induce apoptosis of malignant cells. Anticancer Res 33: 3589-3602, 2013.

17 Bauer G: The antitumor effect of singlet oxygen. Anticancer Res 36: 5649-5664, 2016.

18 Bauer G and Motz M: The antitumor effect of single-domain antibodies directed towards membrane-associated catalase and superoxide dismutase. Anticancer Res 36: 5945-5956, 2016.

19 Bauer G and Graves DB: Mechanisms of selective antitumor action of cold atmospheric plasma-derived reactive oxygen and nitrogen species. Plasma Process Polymers, 2016. doi: 10.1002/ppap.201600089.

20 Scheit K and Bauer G: Direct and indirect inactivation of tumor cell protective catalase by salicylic acid and anthocyanidins reactivates intercellular ROS signaling and allows for synergistic effects. Carcinogenesis 36: 400-411, 2015.

21 Wyllie AH, Kerr JF and Currie AR: Cell death: the significance of apoptosis. Int Rev Cytol 68: 251-274, 1980.

22 Elmore S: Apoptosis: a review of programmed cell death. Toxicol Pathol 35: 495-516, 2007.

23 Bauer G, Bereswill S, Aichele P and Glocker E: Helicobacter pylori protects oncogenically transformed cells from reactive oxygen species-mediated intercellular induction of apoptosis. Carcinogenesis 35: 1582-1591, 2014.

24 Riethmüller M, Burger N and Bauer G: Singlet oxygen treatment of tumor cells triggers extracellular singlet oxygen generation, catalase inactivation and reactivation of intercellular apoptosisinducing signaling. Redox Biol 6: 157-168, 2015.

25 Bechtel W and Bauer G: Modulation of intercellular ROS signaling of human tumor cells. Anticancer Res 29: 4559-4570, 2009.

26 Bauer G: Central signaling elements of intercellular reactive oxygen/nitrogen species-dependent induction of apoptosis in malignant cell. Anticancer Res 37: in press, 2017.

Received November 19, 2016

Revised January 26, 2017

Accepted January 27, 2017 